\author{
Research Paper
}

\title{
Effect of Sand Surface Training on Directed and General Co-contraction of Ankle Joint Muscles During Running
}

\author{
Amir Fatollahi ${ }^{1}$ (i), ${ }^{*}$ Amir Ali Jafarnezhadgero ${ }^{1}$ (1), Saeed Alihosseini ${ }^{2}$
}

1. Department of Sport Management and Biomechanics, Faculty of Educational Sciences and Psychology, University of Mohaghegh Ardabili, Ardabil, Iran 2. Department of Sport Physiology, Faculty of Educational Sciences and Psychology, University of Mohaghegh Ardabili, Ardabil, Iran.

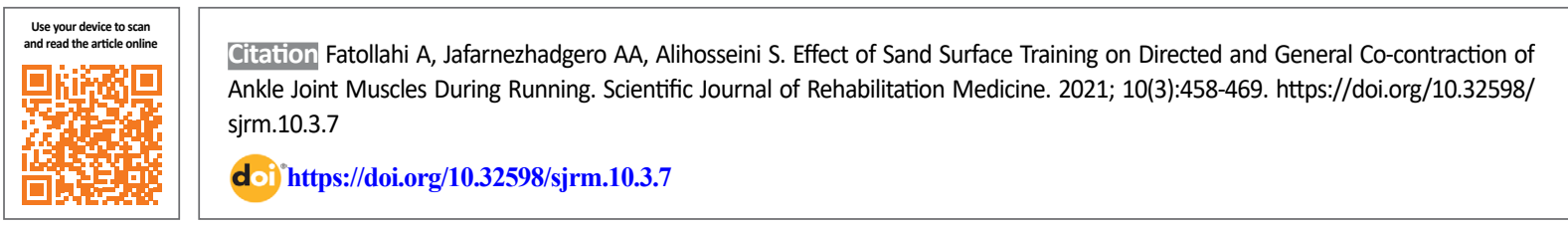

Received: 04 May 2021 Accepted: 16 Jun 2021 Available Online: 23 Jul 2021

Keywords:

Foot overpronation, Sand surface training, Directed cocontraction, General co-contraction

\section{ABSTRACT}

Background and Aims Foot overpronation is one of the most critical factors that cause musculoskeletal injuries and lead to greater mechanical loads in the lower limb structure. Sand is an unstable and unpredictable surface that has particular importance in human movement mechanics. The purpose of this study was to investigate the effect of sand surface training on directed and general co-contraction of ankle joint muscles in individuals with foot overpronation during running.

Methods The present study was a randomized clinical trial type. Statistical samples of the present study included 15 males with foot overpronation in the control group and 15 males with foot overpronation in the intervention group. Individuals in the intervention group performed a sand surface training program consisting of continuous jogging, striding, bounding, galloping, and short sprints for 8 weeks. Tibialis anterior and gas trocnemius medialis muscles activity was evaluated by an 8-channel electromyography system with a surface electrode during running. A two-way ANOVA test was used for statistical analysis at the significant level $P<0.05$. Results The results demonstrated greater ankle-directed co-contraction in the push-off phase during post-test compared with the pre-test in the intervention group during training on sand ( $P=0.040)$. Other components of directed co-contraction in the different stages and general co-contraction in all phases did not demonstrate significant differences after sand training ( $P>0.05)$.

Conclusion It seems greater ankle joint directed co-contraction in the push-off phase after training protocol reduced the risks of lower limb injuries and ankle instability in individuals with foot overpronation in this phase.

\section{Extended Abstract}

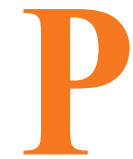

\section{Introduction}

ronation foot is a reversible complex foot deformity comprised of the heel valgus, lower medial longitudinal arch, and abductory twist of the foot. The subtalar joint is the main joint to evaluate pronation foot pathomechanics. The pronated foot is a risk factor for mechanical dysfunctions and injuries in the upper joints such as the ankle, knee, and hip joints. In theory, pronated foot creates internal tibia rotation, hip internal process, knee valgus, and pelvis anterior and ipsilateral tilt. People with pronation feet experience pain and discomfort in the knee joint while walking.

Pronated foot deformity can lead to overuse of the lower limbs, injury, and foot dysfunction, as confirmed by the

\section{* Corresponding Author:}

Amir Ali Jafarnezhadgero, PhD.

Address: Department of Sport Management and Biomechanics, Faculty of Educational Sciences and Psychology, University of Mohaghegh Ardabili, Ardabil, Iran.

Tel: +98 (910) 5146214

E-Mail: amiralijafarnezhad@gmail.com 
navicular drop test. Pronated foot deformity contributes to musculoskeletal injury via the altered motion of the lower extremity. The relationship between foot mobility and lower limb motion was reported, and pronation foot could be recognized as a risk factor for postural stability.

The o-contraction of the muscles provides a means to alter the stability and articular loading of the joint. In general, there are two forms of muscle co-contraction: generalized co-contraction and directed co-contraction. Sand is an unstable and unpredictable surface that has special importance in human movement mechanics. The purpose of this study was to investigate the effect of sand surface training on directed and general co-contraction of ankle joint muscles in individuals with foot overpronation during running.

\section{Methods}

The present study was a randomized clinical trial type. We used the freeware tool G×Power (http://www.gpower. hhu.de/) to calculate a one-sided a priori power analysis with the F test family (ANOVA repeated measures withinbetween interaction) and the respective statistical test based on a related study that examined walking in adults with PF. The power analysis was computed with an assumed Type I error of 0.05 , a Type II error rate of 0.20 (80\% statistical power), two tests (pre, post), a correlation coefficient of 0.5 between observations, and an effect size of 0.70 (i.e., interaction effects) for running. The analysis revealed that 11 participants would be sufficient to observe large Group $\mathrm{x}$ Time interactions.

For both groups, participants were recruited if they showed a navicular drop of more than $10 \mathrm{~mm}$, and a foot posture index larger than 10 . Of note, the navicular drop was measured as the difference in navicular height during non-weight bearing compared with full weight bearing of the foot during quiet unilateral standing. Also For both groups, a priori defined exclusion criteria comprised a history of musculoskeletal surgery at the trunk and/or lower limbs, neuromuscular or orthopedic disorders (except of over Pronated Feet (PF)), limb length differences larger than $5 \mathrm{~mm}$, and in control group the performance of strenuous physical exercises $\leq 8$ weeks prior to testing. The procedures were explained to the participants prior to obtaining informed consent in accordance with the Declaration of Helsinki. The included participants reported demographic information including: age, limb dominance, and injury history. All participants were right footed and right handed as determined by a kicking and throwing ball test.

Statistical samples of the present study included 15 males with foot overpronation in the control group and 15 males with foot overpronation in the intervention group. Individuals in the experimental group performed a sand running training program consisting of walking, continuous jogging, striding, bounding, galloping, and short sprints for eight weeks (3 sessions per week). Every session was started with a warm-up and stretching session for five minutes and was ended with a cool-down session for five minutes. The total training duration was 50 minutes per session. Individuals in the control group did not perform any exercise and were re-evaluated after 8 weeks. All participants were asked not to participate in any other sports and activities during the intervention period.

Tibialis anterior and gastrocnemius medialis muscles activity was evaluated by an 8-channel electromyography system with a surface electrode during running. The raw EMG signals were digitized at $2000 \mathrm{~Hz}$ and streamed via Bluetooth to a computer for further analysis. According to the European recommendations for surface electromyography (SENIAM), the skin surface was shaved and cleaned with alcohol $\left(70 \%\right.$ Ethanol- $\left.\mathrm{C}_{2} \mathrm{H}_{5} \mathrm{OH}\right)$ over the selected muscles. After that, the skin was scratched gently prior to electrode placement. Electromyography (EMG) data were synchronized using Nexus software (Oxford Metrics, Oxford, UK). All analyses were performed using SPSS v. 20. Normal distribution was examined and confirmed using the Shapiro-Wilk Test. A two-way ANOVA test was used for statistical analysis at the significant level $\mathrm{P}<0.05$.

\section{Results}

No significant between-group differences were found at baseline for all examined variables while running $(\mathrm{P}>0.05)$. Statistically significant main effects of "time" were found for directed co-contraction dorsiflexion/plantar flexion of ankle joint muscles $(\mathrm{P}=0.040)$ during the push-off phase. Pair-wise comparisons revealed significantly greater directed co-contraction dorsiflexion/plantar flexion of ankle joint muscles in the post-test compared with the pre-test during running.

The results did not demonstrate any significant main effects of "time" for directed co-contraction dorsiflexion/ plantar flexion of ankle joint muscles $(\mathrm{P}>0.05)$ during loading response, mid-stance, and swing phases.

The results did not demonstrate any significant main effects of "group" for directed co-contraction dorsiflexion/ plantar flexion of ankle joint muscles $(\mathrm{P}>0.05)$ during loading response, mid-stance, push off and swing phases. Also, general co-contraction of ankle joint muscles $(\mathrm{P}>0.05) \mathrm{did}$ not demonstrate any significant main effects of "group" for loading response, mid-stance, push off and swing phases during training on sand. The results did not show signifi- 
cant group-by-time interactions for directed co-contraction dorsiflexion/plantar flexion of ankle joint muscles $(\mathrm{P}>0.05)$ for loading response, mid-stance, push off and swing phases during running. Also, results did not demonstrate any significant main effects of the group by time interactions for general co-contraction of ankle joint muscles $(\mathrm{P}>0.05)$ during loading response, mid-stance, push off and swing phases of running.

\section{Discussion and Conclusion}

It seems greater ankle joint directed co-contraction in the push-off phase after training protocol reduced the risks of lower limb injuries and ankle instability in individuals with foot overpronation in this phase.

\section{Ethical Considerations}

Compliance with ethical guidelines

The ethics committee approved the research protocol of Ardabil University of Medical Sciences (Code: IR.ARUMS.REC.1398.484) and was registered with Iran's Clinical Trial Code (Code: RCT20191211045704N1). All research was conducted following the Helsinki Declaration.

Funding

This study was extracted from the of MSc. thesis of first author at the Department of Physical Education of Ardabil University of Medical Sciences.

Authors' contributions

Authors contributed equally in preparing this article.

\section{Conflict of interest}

The authors declared no conflict of interest.

\section{Acknowledgments}

We would like to thank Mohaghegh Ardabili University for its financial support. 
مقاله بزووهشى

اثر تمرين روى سطح شن بر همانقباضى جهتدار و عمومى عضلات مفصل مج يا طى دويدن امير فتحاللهى' ــ، "اميرعلى جعفرنزاد كرو' ه، سعيد على حسينى'

1. ا.كروه مديريت و بيومكانيك ورزشى، دانشكده علوم تربيتى وروانشناسى، دانشعاه محقق اردبيلى، اردبيل، ايران.

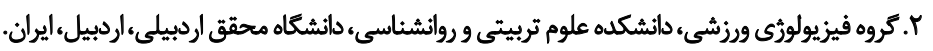

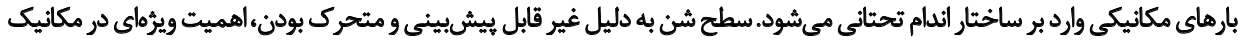

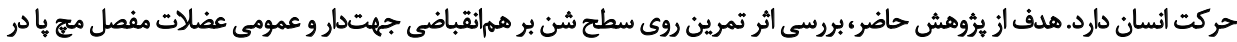

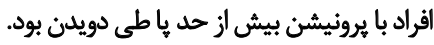

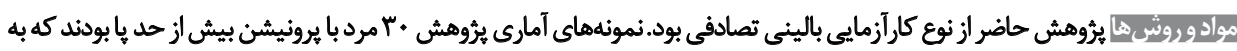

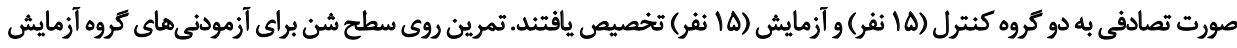

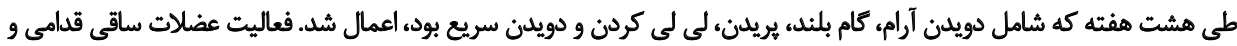

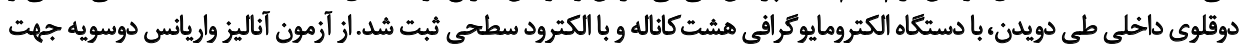

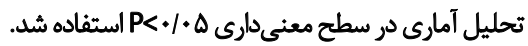

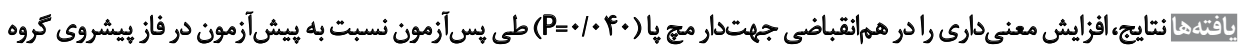

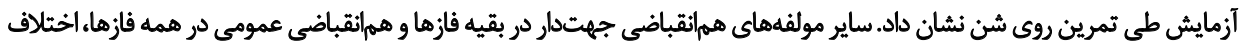

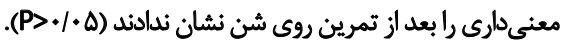

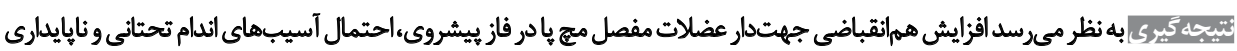

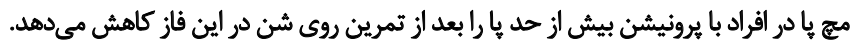

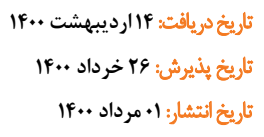

كانيدوإlog

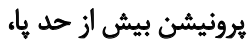

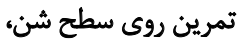

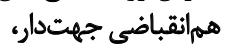
هم|نقباضى عمومى جهي

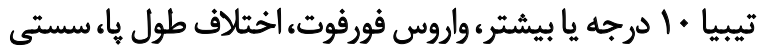

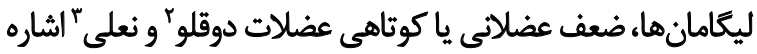

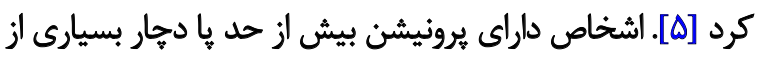

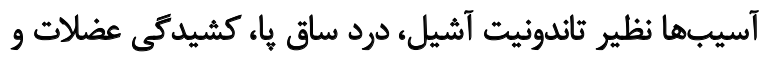

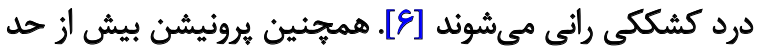

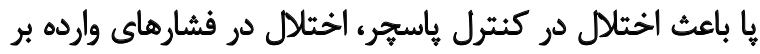

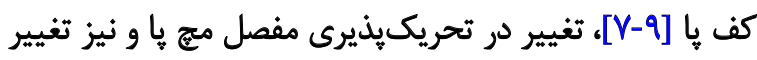

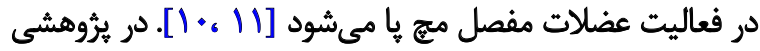

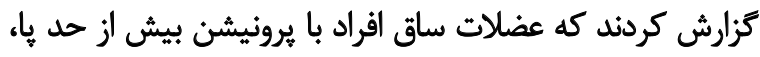

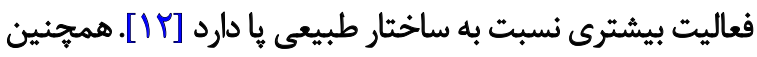

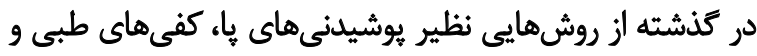

ازويلن يكى از عمومىترين حركات انسان است كه در بسيارى

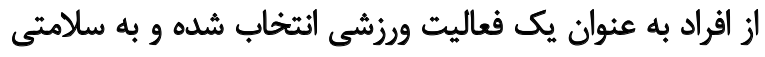

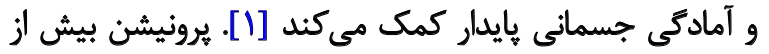

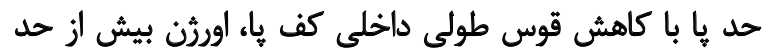

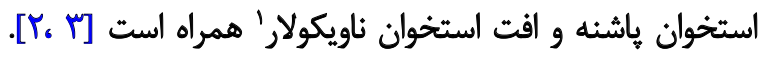

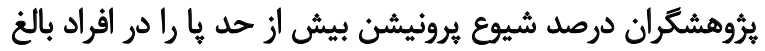

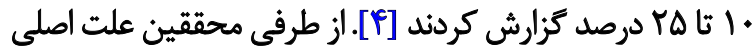

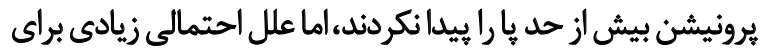

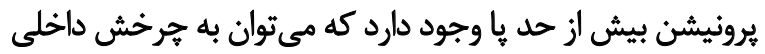


بررسى كردند كه به ترتيب افزايش زاويه فلكسورى مفاصل ران و

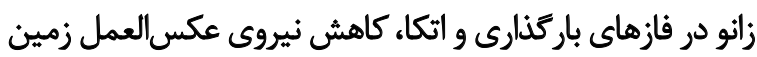

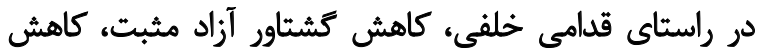

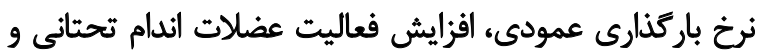

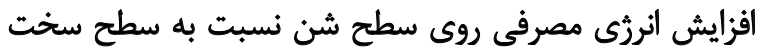

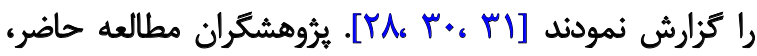

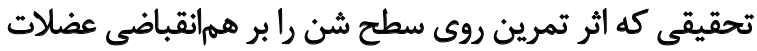

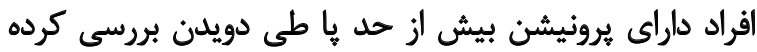

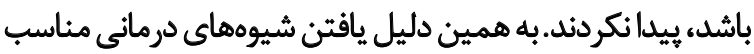

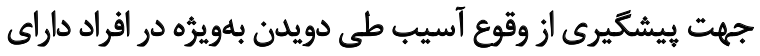
يرونيشن بيش از حد ها و بهبود اين عارضه در اين افرين إداد ضرورت

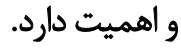

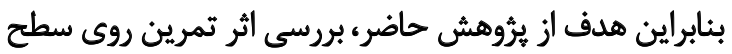

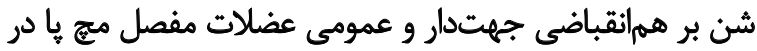

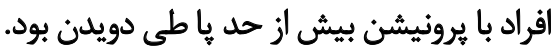

\section{lo.}

يروهش حاضر از نوع كارآزمايى بالينى تصادفي بود. نرمافزار

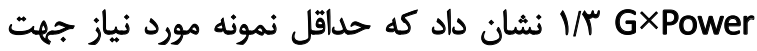

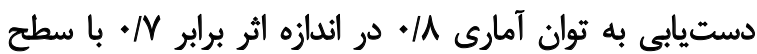

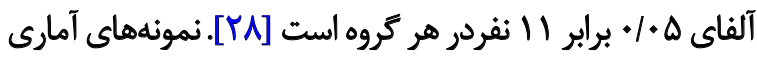

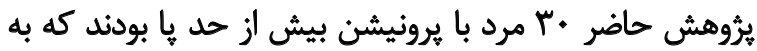

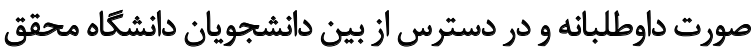

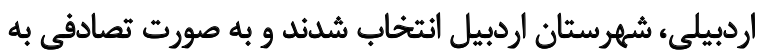

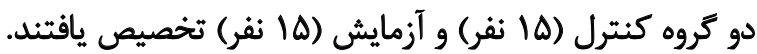

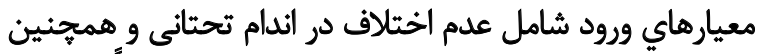
عدم سابقه ابتلا به آسيبهاي اسكلتى عضلاني اختي خصوصأ در ناحيه

اندام تحتاني بود.

تمرين روى سطح شن [جr] براى آزمودنىهاى كروه آزمايش

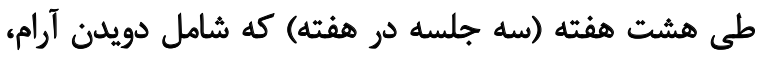

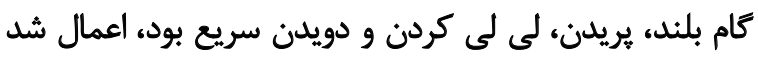

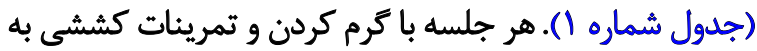

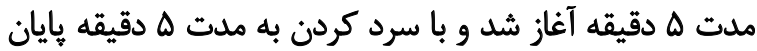

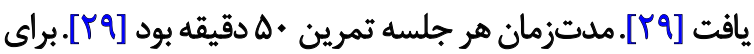

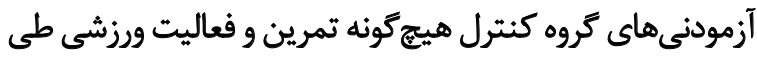
هشت هفته اجراى يرؤوهش اعمال نشد.

از روش افتادكى استخوان ناوي براي تشخيص آزمودنى هاي

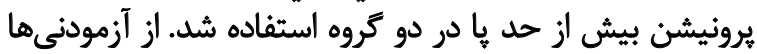

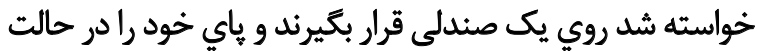

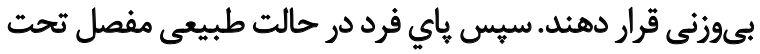

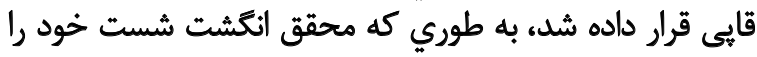

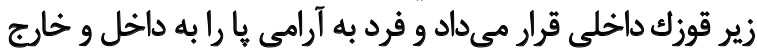

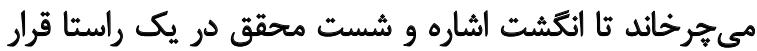

تييّينك براى درمان برونيشن بيش از حد إيا استفاده شده است

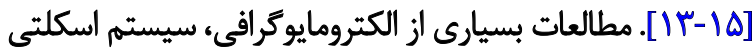

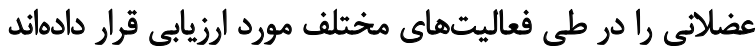

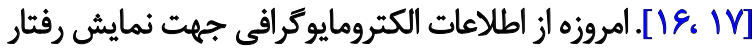

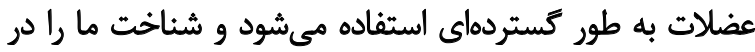

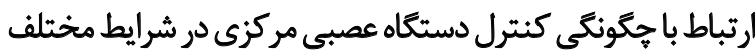

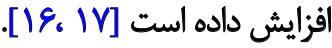

همانقباضى عضلانى به عنوان فعاليت همزمان عضلات مختلف

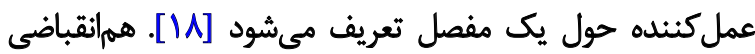

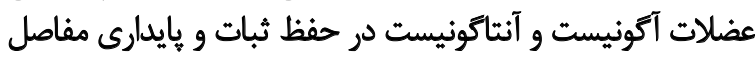

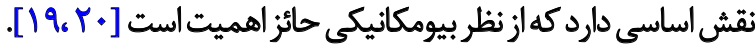

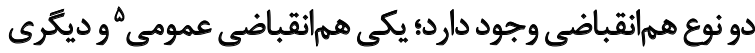

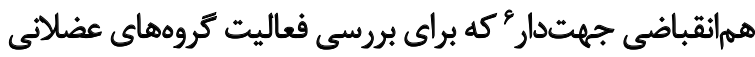

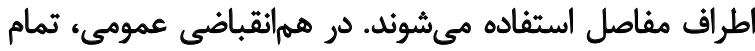

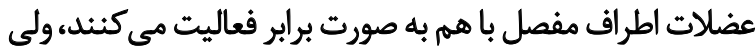

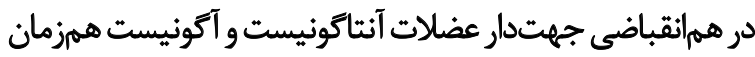

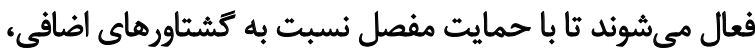

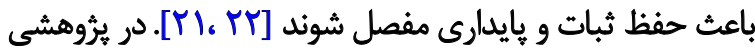

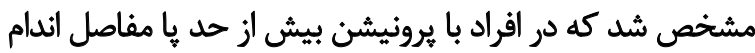
تحتانى داراى بى ثباتى و تحريكي

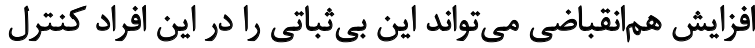

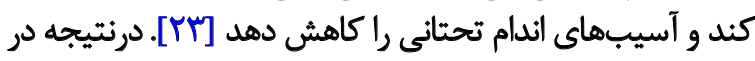

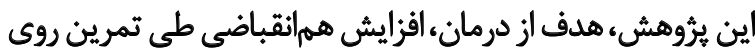

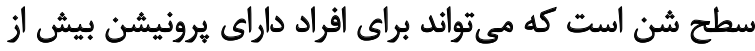

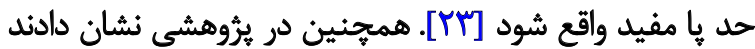

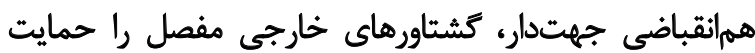

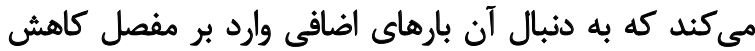

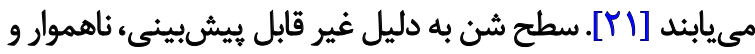

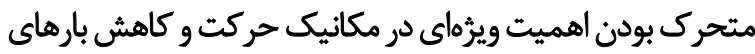

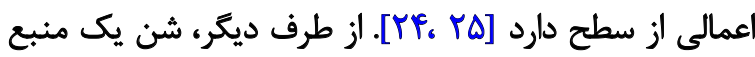

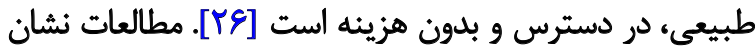

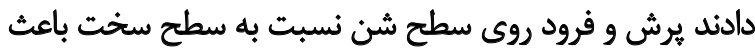

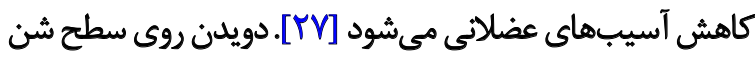

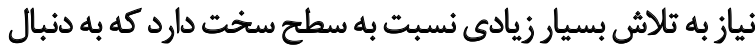

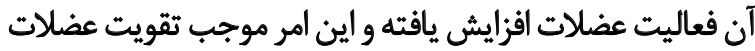

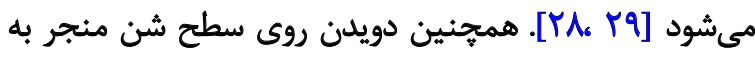

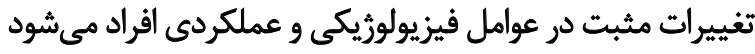

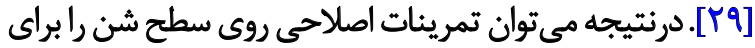

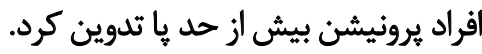
مطالعات بيشين اثر آنى راه رفتن روى سطح شن بر كينماتيك، كينتيك و فعاليت الكتريكى عضلات و ميزان انرثى مصرفى راهن رينانيا

\footnotetext{
4. Co-contraction

5. General co-contraction

6. Directed co-contraction 
جدول ا. بروتكل تمرين روى سطح شن كروه آزمايش

\begin{tabular}{|c|c|c|c|c|c|}
\hline ريكاورى (دقيقه) & مسافت اجراي تمرين (متر) & تكرار تمرين & مدت تمرين (دقيقه) & نوع تمرين & شماره \\
\hline - & Q. & - & r. & دويدن آرام & 1 \\
\hline 1 & $\Delta$. & rt & $r$ & كام بلثد & r \\
\hline 1 & r. & rكا & $r$ & يبريلن & $r$ \\
\hline 1 & r. & r & r & لى لى كردن & r \\
\hline$r$ & ro & " & 8 & دويلن سريع & $\Delta$ \\
\hline
\end{tabular}

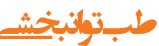

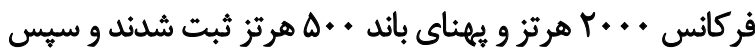

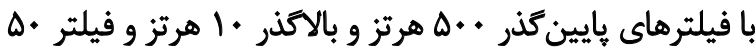

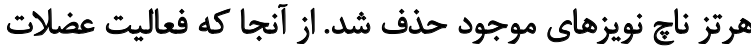

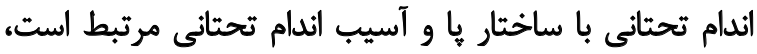

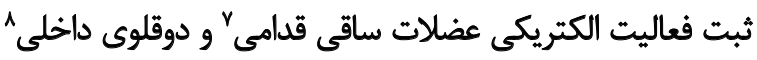

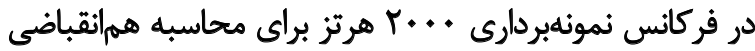

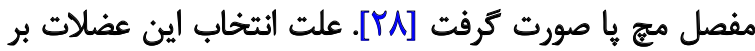

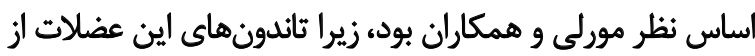

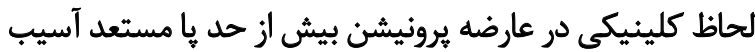

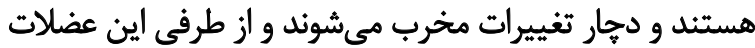

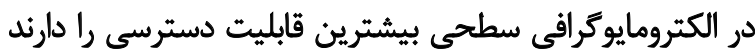

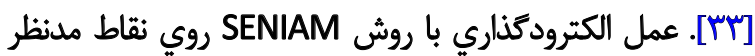

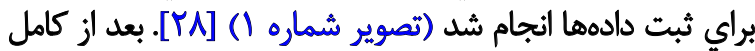

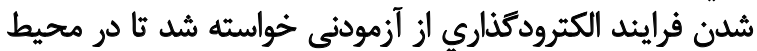

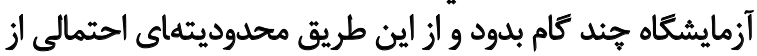

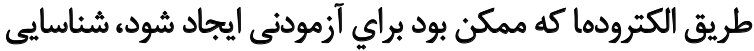

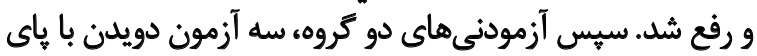

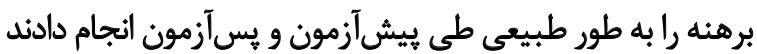
كه ميانكين اين سه تكرار براى تجزيه و تحليل دادهها استفاده شد.

براي تجزيه و تحليل دادهایى حاصل از الكترومايوكرافى، از ماز

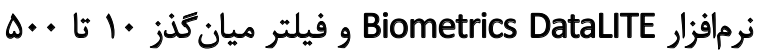
هرتز استفاده شد.براى نرمال كردن سيكنال هاى الكترومايوگر افيى،

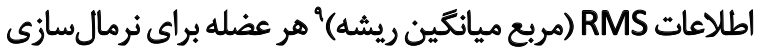

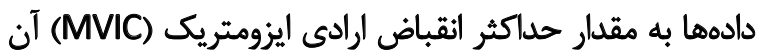

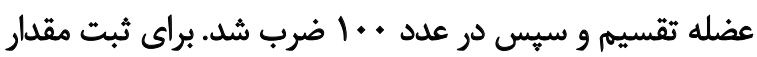

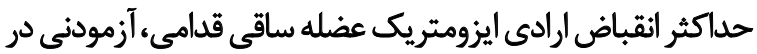

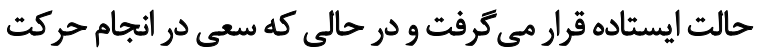

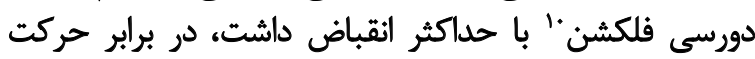

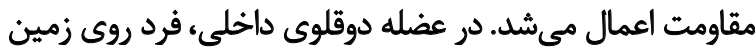

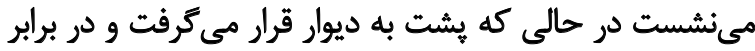

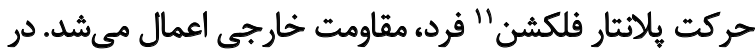

7. Tibialis anterior

8. Gastrocnemius medialis

9. Root Mean Square (RMS)

10. Dorsiflexion

11. Plantar flexion

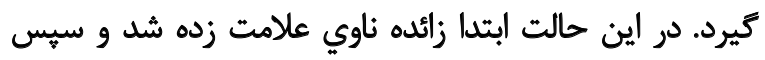

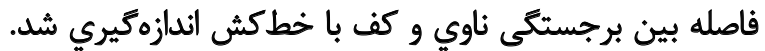

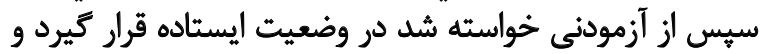

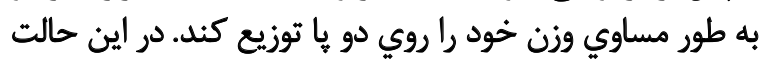

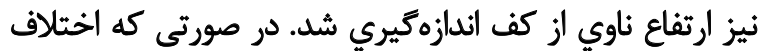

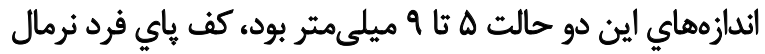

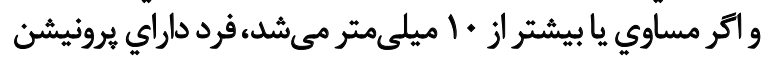

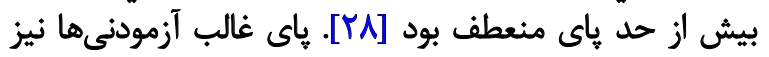

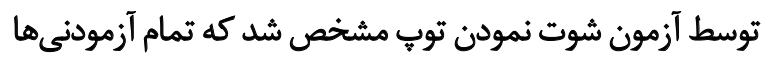

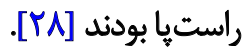

يروتكل تحقيق توسط كميته اخلاق دانشعاه علوميزشكى

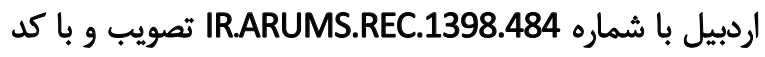
كارآزمايى بالينى ايران به شماره تئماره

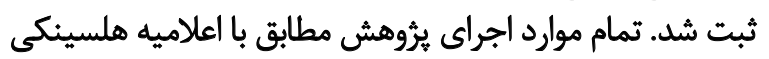

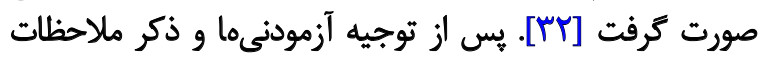

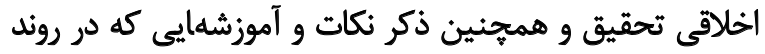

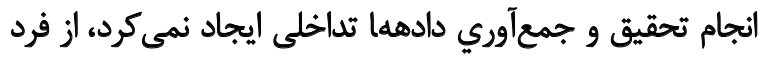

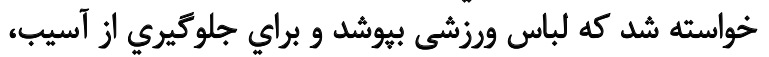

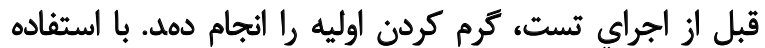

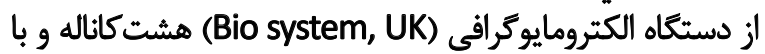

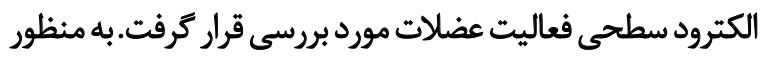

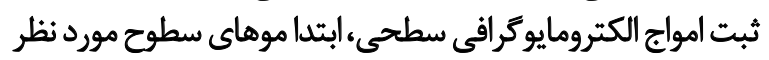

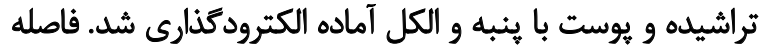

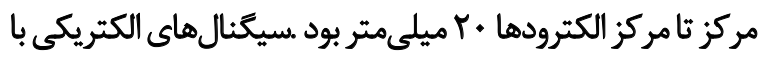

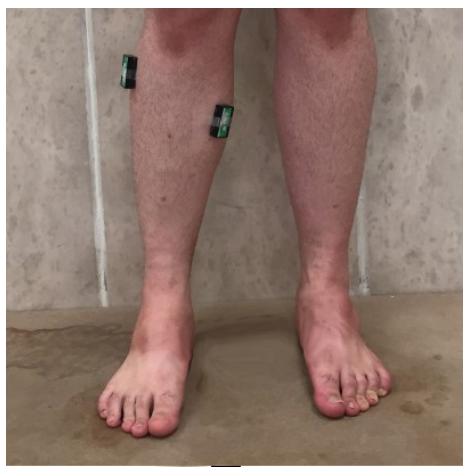


را در همانقباضى جهتدار دورسىفلكسورى خيلانتارفلكسورى

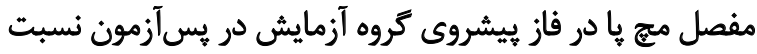

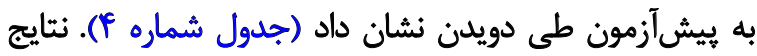

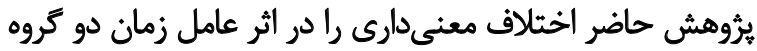

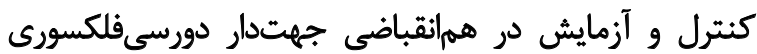

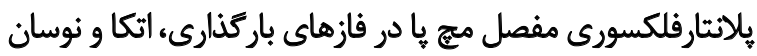

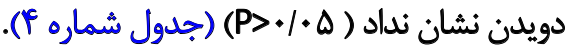
نتايج يثروهش حاضر اختلاف معنىدارى بين دو كروه

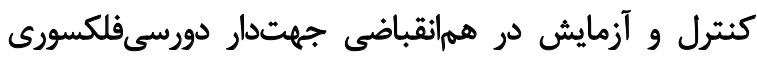

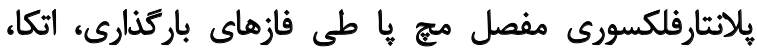

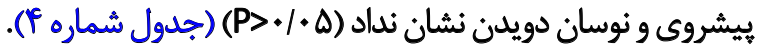

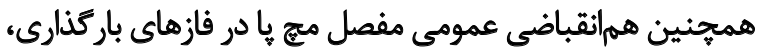

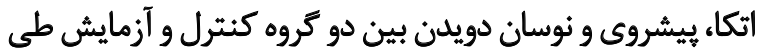

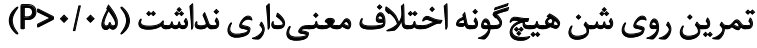

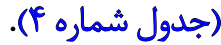

با توجه به نتايج يُروهش حاضر، در بررسى اثر تعاملى زمان و و

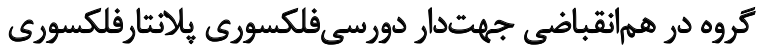

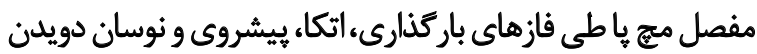

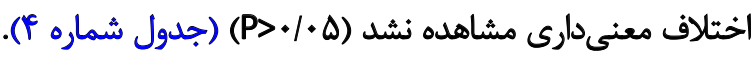

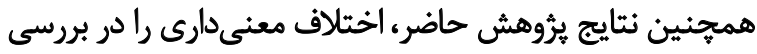

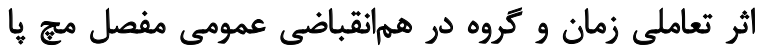

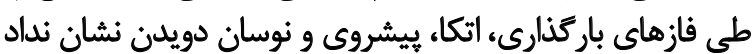

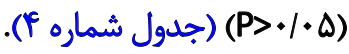

ب ب

هدف از يُوهش حاضر، بررسى اثر تمرين روى سطح شن بر بر براف

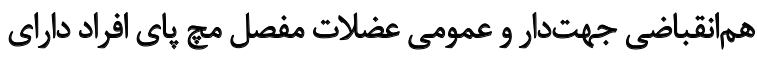

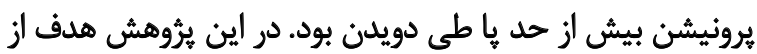

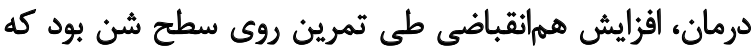

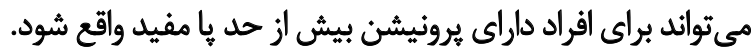

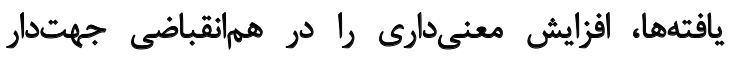

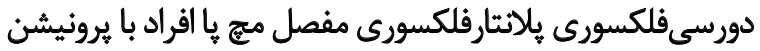

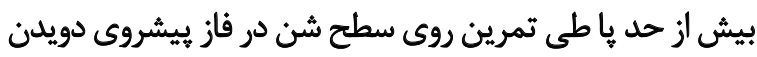

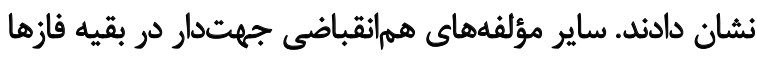

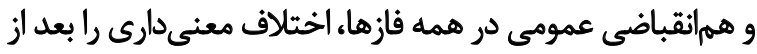

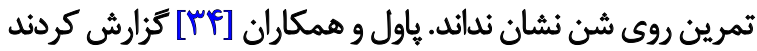

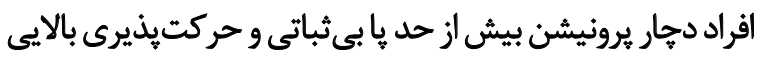

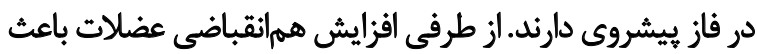

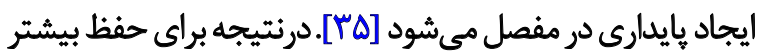

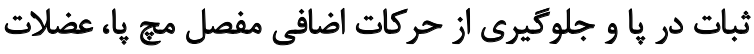

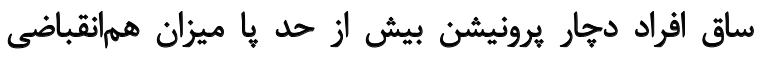
بيشترى رانياز دارند كه بانتايج تحقيق حاضر همسوست.
اين حالت از وى خواسته شد تا حداكثر تلاش خود را براي ايجاد حركت يلانتار فلكشن به كار كيرد.

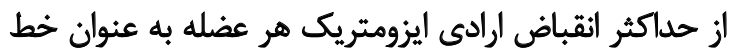

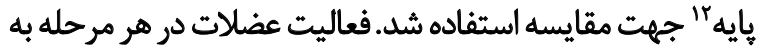

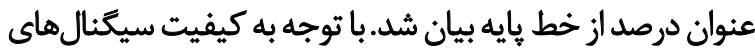

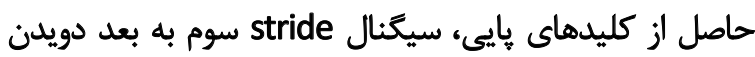

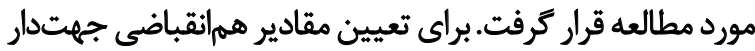

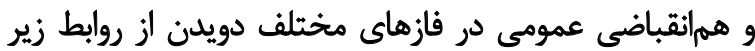

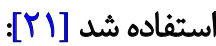

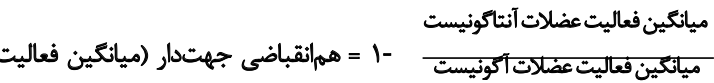

$$
\begin{aligned}
& \text { عضله آنتاكونيست >ميانكين فعاليت عضله آكونيست) }
\end{aligned}
$$

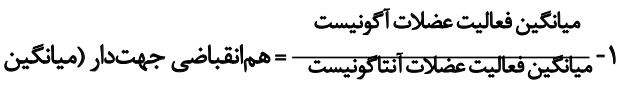

$$
\begin{aligned}
& \text { فعاليت عضله آنتاكونيست>ميانكين فعاليت عضله آكونيست) } \\
& \text { همرانقباضى عمومى=مجموع ميانكين فعاليت تمام عضلات مفصل }
\end{aligned}
$$

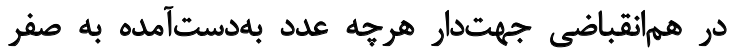

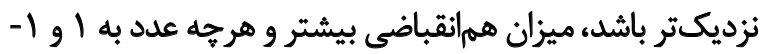

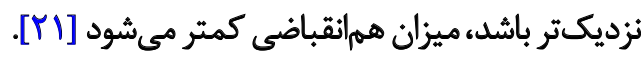

تجزيه و تحليل دادهها از طريق نرمافزار SPSS نسخه · ب انجام

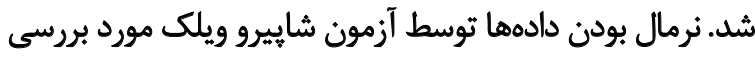

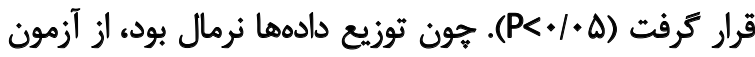

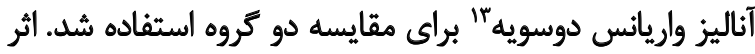

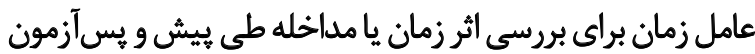

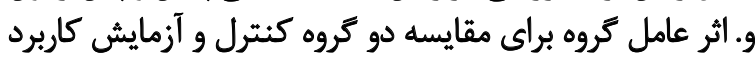

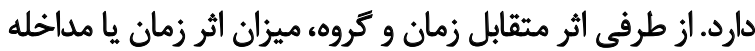

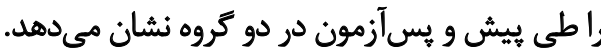

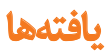

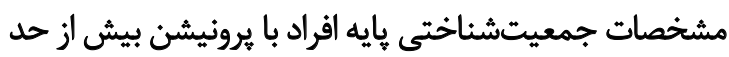

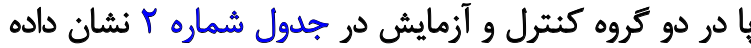

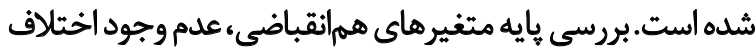

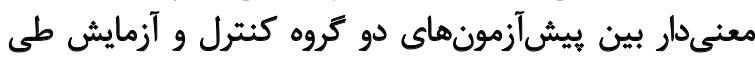

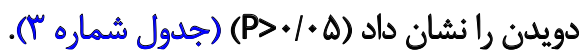

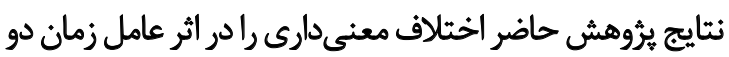

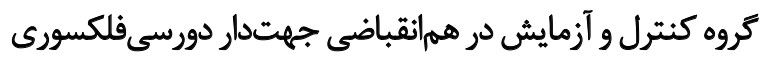

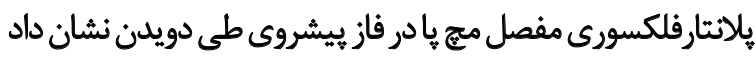

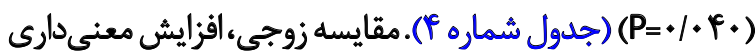


جدول r. مقايسه مشخصات جمعيتشناختى دو كروه كنترل (n=10) و آزمايش (n=10)

\begin{tabular}{|c|c|c|c|}
\hline \multirow{2}{*}{ سطح معنى اري } & \multicolumn{2}{|c|}{ ميانكين +انحراف استاندارد } & \multirow{2}{*}{ مشخصات جمعيتشناختى } \\
\hline & مروه آزمايش & كروه كثترل & \\
\hline.$/ 29$ & 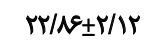 & $r r / T E \pm T / M r$ & سن (سال) \\
\hline ( & $I V \in / V \varepsilon \pm V / M r$ & $I N / V F \pm N T Y$ & قلد (سائتى مثر) \\
\hline.$/ 8 I T$ & $n / q+ \pm|1 / 8|$ & $v / \pi+ \pm 11 / M$ & جرم بلن (كيلوكرم) \\
\hline .1919 & $\pi \in / T \pm \pm r / q Y$ & $r \in / r A \pm r / R q$ & شاخص توده بدنى (كيلوكرم بر متر مريع) \\
\hline
\end{tabular}

نتايج بثروهش حاضر افزايش معنى دارى را در همانقباضى

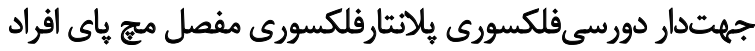

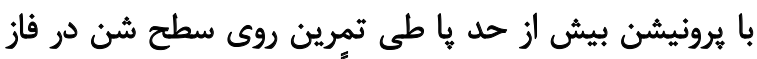

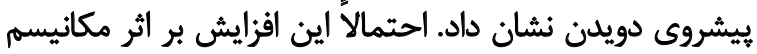

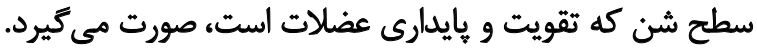

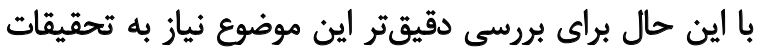

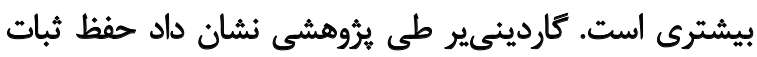

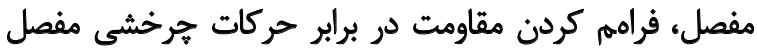

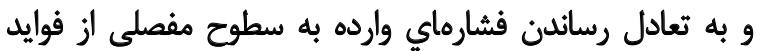

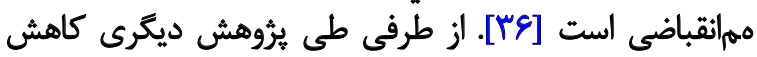

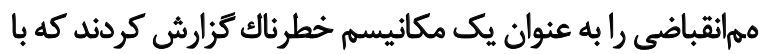

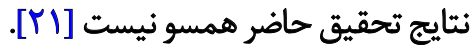

نتايج يروهش حاضر با توجه به مطالب ذكرشده، افزايش

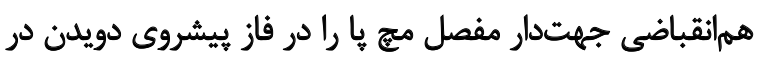

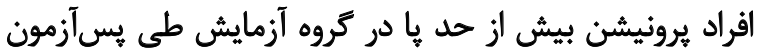

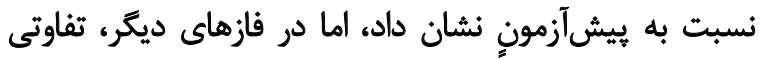

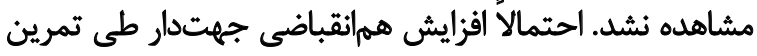

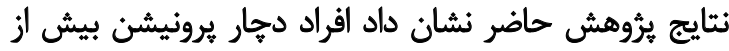

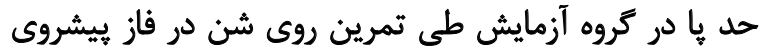

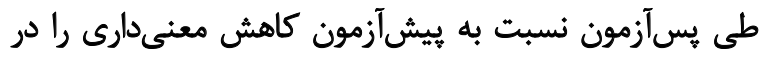

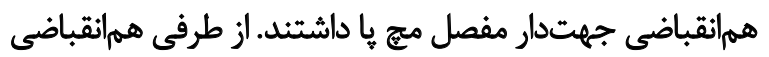

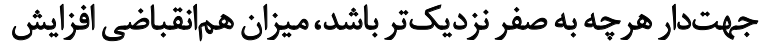

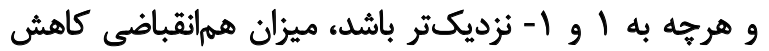

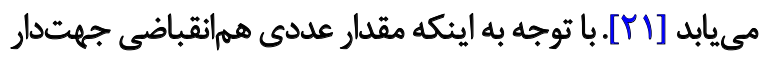

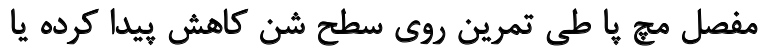

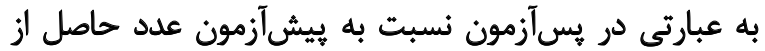

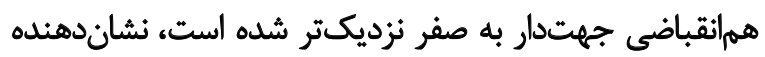

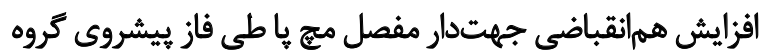

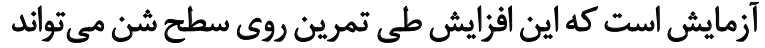

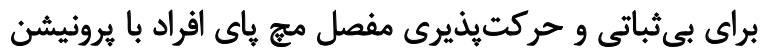

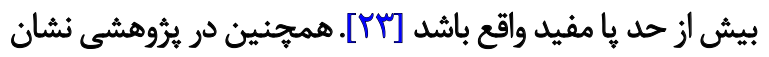

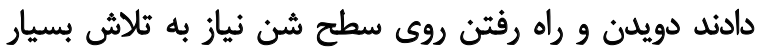

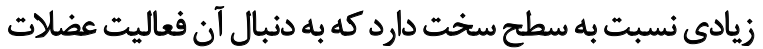

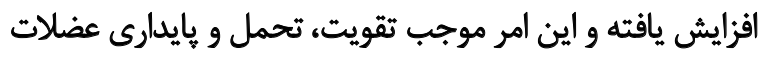

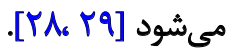

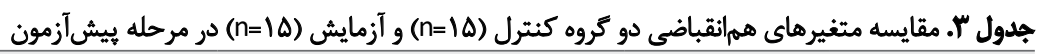

\begin{tabular}{|c|c|c|c|c|}
\hline \multirow{2}{*}{ سطح معنى دارى } & \multicolumn{2}{|c|}{ مياتكين+انحراف استاندارد } & \multirow{2}{*}{\multicolumn{2}{|c|}{ متغيرها }} \\
\hline & كروه آزمايش & كروه كثترل & & \\
\hline . Nar & $T T / N \cdot \pm \mid r / \Delta T$ & $r \notin / V \Lambda \pm q / \mp A$ & فاز باركنارى (LR) & \\
\hline.$/ \Delta A T$ & $V R / T V \pm T \Delta / \Delta T$ & $E Q \Delta Q T \pm M / \cdot Y$ & فازي اتكا (MS) & همرانتباضى عمومى \\
\hline - MPra & $8 \pi / 19 \pm 18 / * 1$ & $8 V / V+1 \% / p H$ & فاز ييشروى (PO) & : (GCC) \\
\hline .rif & $r \cdot M I \pm I V / T$ & $\pi / \Delta \cdot \pm 1 \cdot / / V$ & فاز نوسان (SW) & \\
\hline.$/ 94 y$ & $-\cdot / r q \pm \cdot / 11$ & $-\cdot / 0 \cdot \pm \cdot / 1 V$ & فاز باركذارى (LR) & \\
\hline MIE & $-\cdot / Q \cdot \pm \cdot / r V$ & $-\cdot / 4 \varepsilon_{ \pm} \cdot / M$ & فاز اتكا (MS) & جهتدار \\
\hline . Nar & $.189 \pm+/ r$ & $.|8 \Lambda \pm+| M \mid$ & فاز ييشروى (PO) & (DCC) \\
\hline .MPr & $. / 14 \pm \pm / 16$ & $\cdot|8 x \pm \cdot / 1|$ & فُازز نوسان (SW) & \\
\hline
\end{tabular}


جدول f. ميانكين و انحراف استاندارد متغيرهاى همانقباضى بين دو كروه كنترل (n=1ه) و آزمايش (D=10) طى تمرينات روى شن

\begin{tabular}{|c|c|c|c|c|c|c|c|c|c|c|}
\hline \multicolumn{9}{|c|}{ مياتكين+|تحرافسمعيار } & \multirow{3}{*}{\multicolumn{2}{|c|}{ مثثيرها }} \\
\hline \multicolumn{3}{|c|}{ سطح معنى دارى } & \multicolumn{3}{|c|}{ كروه آزمايش } & \multicolumn{3}{|c|}{ كروه كتترل } & & \\
\hline زمان و وثروه & اثر عامل & زاثر عامل & اثدازه اثر & يسآزمون & ييش أزمون & اثدازه & يسآزمون & ييشأزمون & & \\
\hline$. / T A \mid$ & - IMF &.$/ m n$ &.$/ M$ & $E g / E D \pm I F$ & $M T / V= \pm I Y / \Delta r$ & $\% v$ & $P \in / N E \pm N \in \Lambda$ & $P F / V A \pm V / A$ & فاز باركنارى (LR) & \\
\hline .940 &.$/ \mathrm{TMA}$ & r &.$/ N$ & $W / r A \pm r+/ I T$ & $r=/ r Y \pm r \Delta / \Delta T$ & $.1 . r$ & $89 / 10 \pm 10 / 9$. & $S Q / \Delta T \pm T r / \cdot r$ & فاز اتكا (MS) & $\mathrm{GCC}$ \\
\hline.$/ \mathrm{Vr}$ &.$/ T A F$ &.$/ 4 \cdot 8$ &.$/ 19$ & $8 \cdot / V A \pm V / P F$ & $9 / / 1 q \pm 18 /+1$ & $.1+1$ & $s v / r f \pm 1 r / \Delta$ & $s V / M Y \pm 1 F / r r$ & فاز ييشروى (PO) & 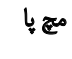 \\
\hline . Isar &.$/ \pi$ &.$/ 9 n$ & $\% \vee r$ & $r N Q I \pm 1 F / F \Delta$ & $f \circ / 11 \pm I V / M T$ &.$/ r$ & $n+q \cdot \pm 1 \cdot / 41$ & $\Pi / \Delta \cdot \pm 1 \cdot / N \gamma$ & فاز توسان (SW) & \\
\hline .1891 & - /Ara &.$/ 999$ & $\% A$ & $-\cdot / 1 \Delta \cdot \pm * 1 \cdot 9$ & $-* / q q \pm \cdot / 11$ &.$/ r$ & $-* / 4 A \pm+118$ & $-\cdot / 10 \cdot \pm \cdot / 1 Y$ & قاز باركذارى (LR) & \\
\hline$\cdot M r$ & . $|A \Delta|$ & . NET & .1 .0 & $-+/ 19 \pm \pm / 18$ & $-* / \Delta \cdot \pm+/ T V$ &.$M$ & $-+/ 4 q \pm+/ \% q$ & $-. / 48 \pm+/ 11$ & فاز اتكا (MS) & DCC \\
\hline.$/ M A$ & ./Arq & $1 . \varphi^{\circ}$ & - par & $\cdot|8| \pm * / 1 \mid$ & $. / 89 \pm / M$ &.$/ N$ & $.188 \pm+110$ & $\cdot|F A \pm \cdot| M \mid$ & فاز ييشروى (PO) & 4 \\
\hline . /arq & .1114 & - mar &.$/ M$ & $\cdot / \Delta \Delta \pm \cdot / M$ & $\cdot / M \pm \pm / 11 F$ & $\% v$ & $.194 \pm=119$ & $. / 84 \pm \cdot / 11$ & فاز نوسان (SW) & \\
\hline
\end{tabular}

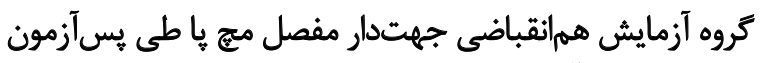

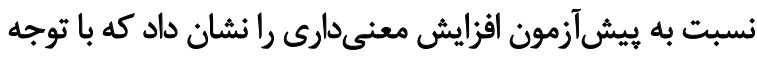

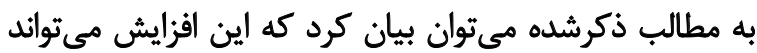

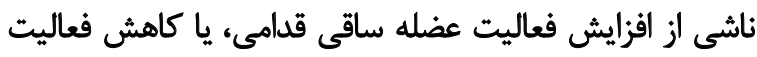

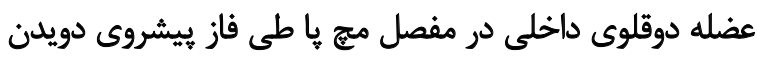

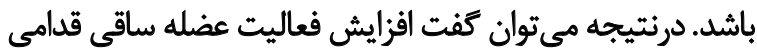

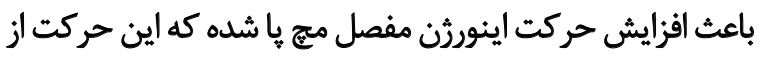

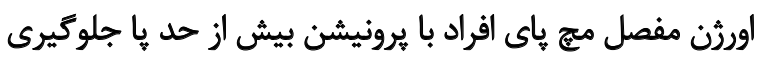

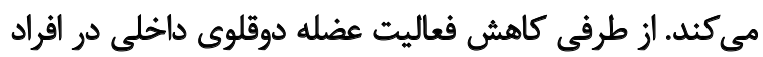

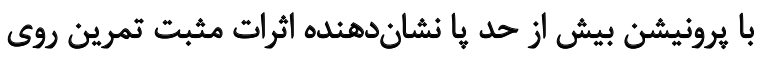

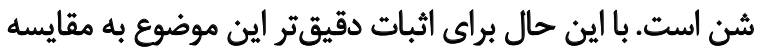

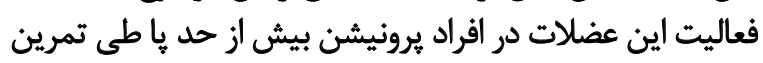

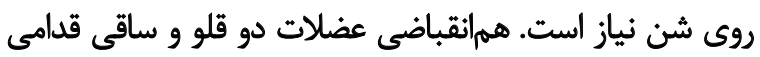

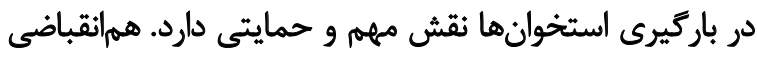

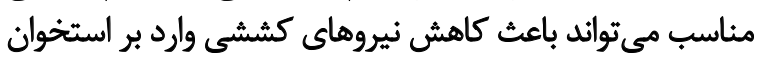

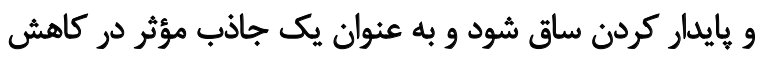

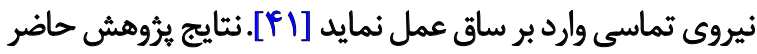

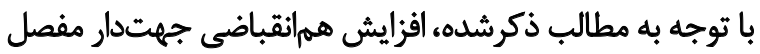

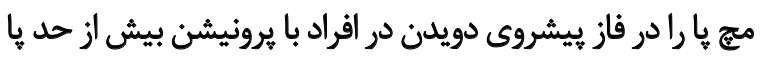

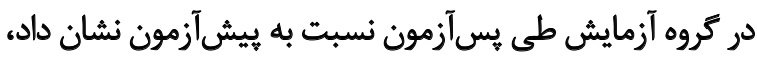

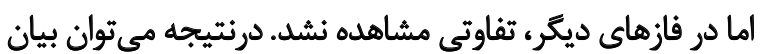

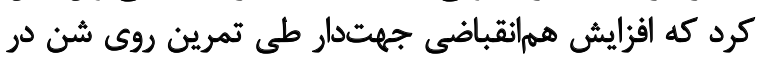

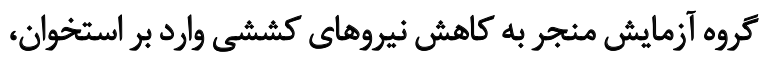

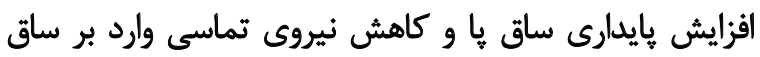

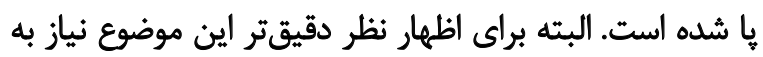
تحقيقات بيشترى است.

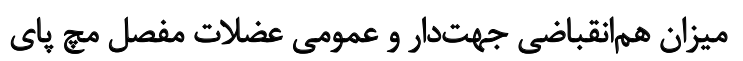

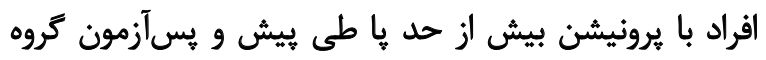

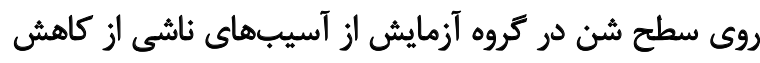

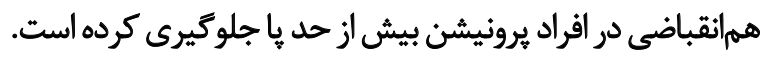

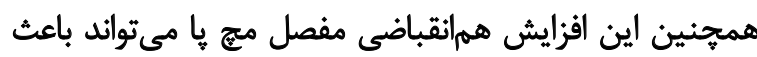

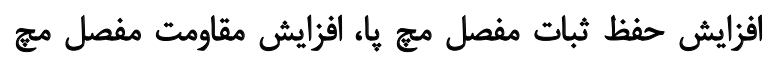

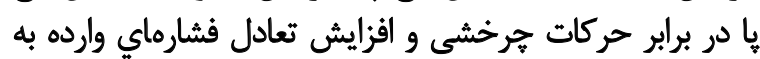

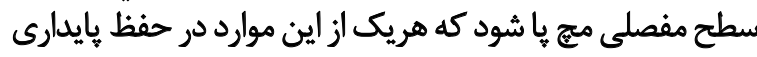

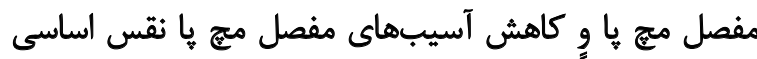

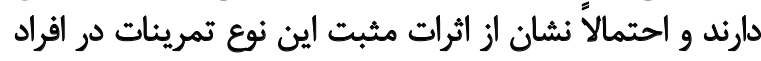

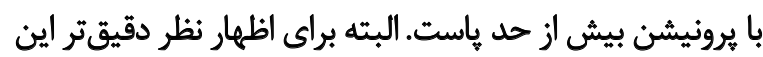

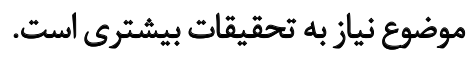

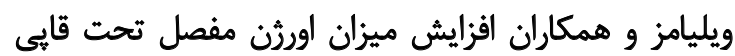

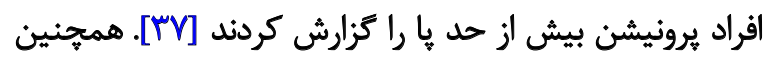

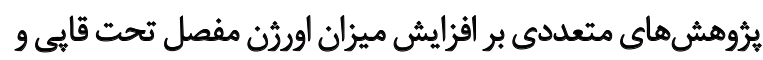

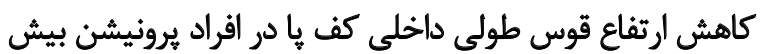

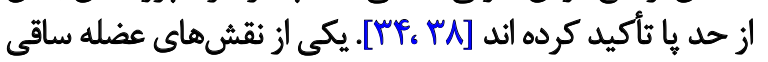

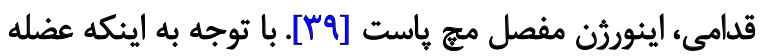

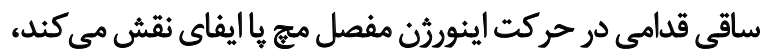

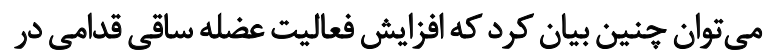

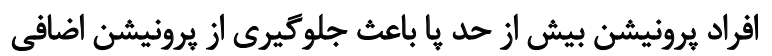

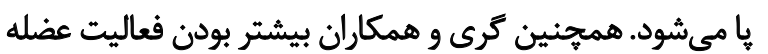

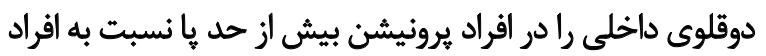

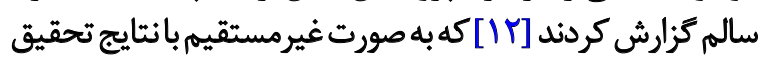

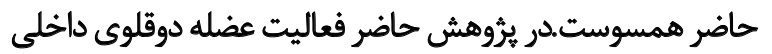

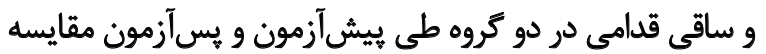

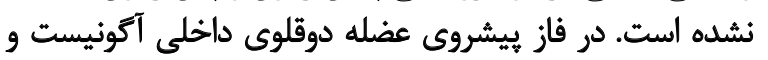

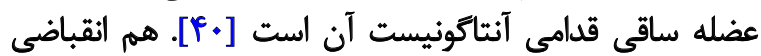

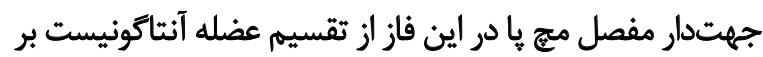

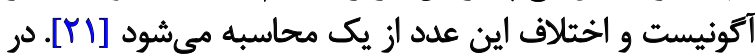




$$
\text { تعارض مثاقع }
$$

بنابر اظهار نويسندكان اين مقاله تعارض منافع ندارد.

$$
\text { تشكر وقرداني }
$$

از دانشكاه محقق اردبيلى براي حمايتهاي مالى تشكر و

$$
\text { قدرداني ميشود. }
$$

آزمايش در فازهاي باركذاري، اتكاو نوسان دويدن اختلاف معنى دارى

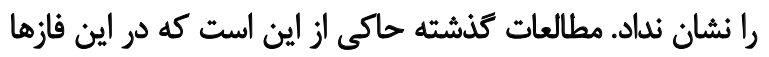

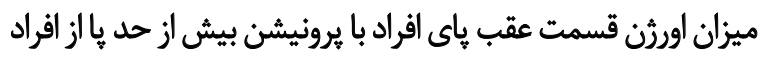

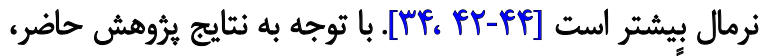

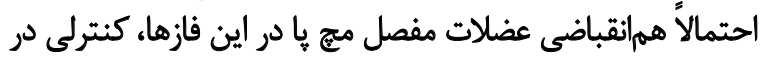

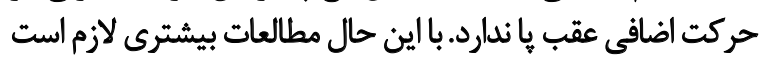

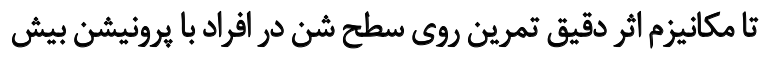
از حد با مورد ارزيابى قرار تيرد.

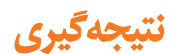

نتايج اين مطالعه نشان داد همانقباضى جهت بدار مفصل مج ئ بادر

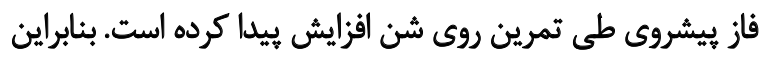

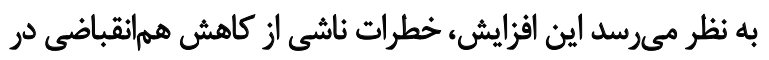

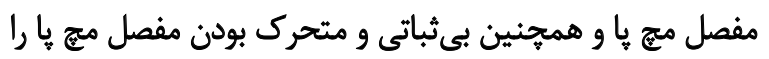

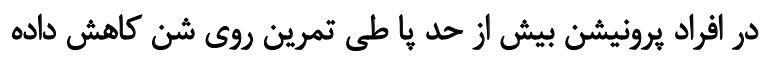

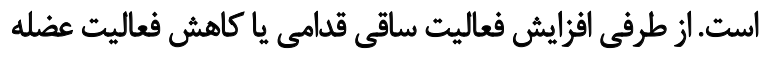

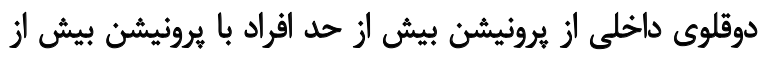

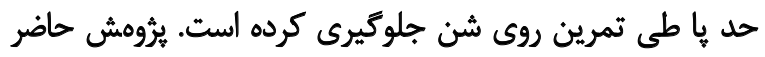

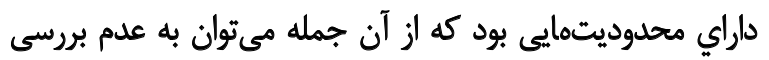

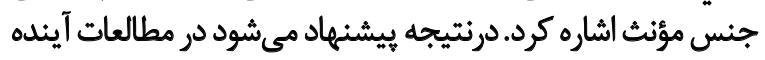

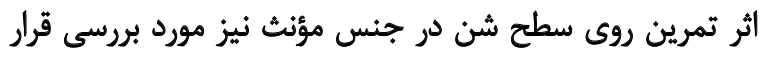

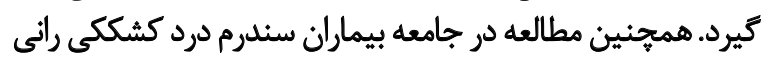

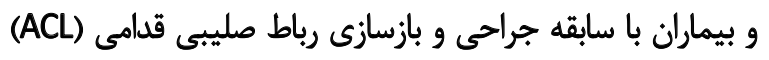

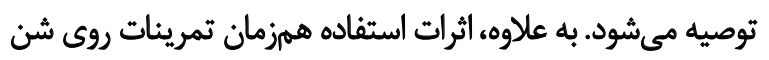

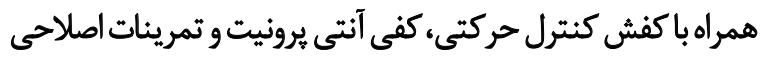

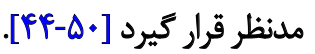

مالامظات الخاقي

\section{ييروى أز اصول اخلاق يروهش}

بروتكل تحقيق توسط كميته اخلاق دانشكاه علوميزشكى اردبيل

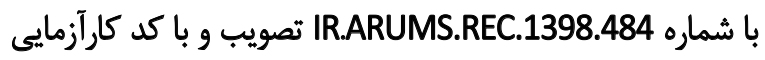

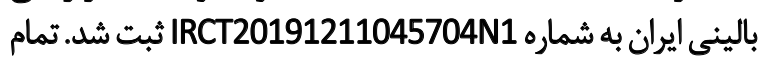

موارد اجراى يرُوهش مطابق با اعلاميه هلسينكى صورت كرفت.

$$
\text { حامي مالى }
$$

اين مقاله بركرفته از هاياننامه كارشناسى نويسنده اول در تروه

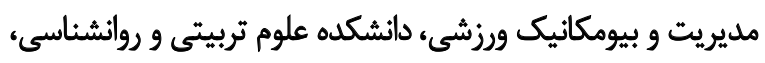
دانشعاه محقق اردبيلى، اردبيل است.

مشاركت نويسندكان تمام نويسندكان در آمادهازى اين مقاله مشاركت يكسان داشته 


\section{References}

[1] Van Gent BR, Siem DD, van Middelkoop M, van Os TA, Bierma-Zeinstra SS, Koes BB. Incidence and determinants of lower extremity running injuries in long distance runners: A systematic review. British Journal of Sports Medicine. 2007; 41(8):469-80. [DOI:10.1136/ bjsm.2006.033548] [PMID] [PMCID]

[2] Prentice WE. Rehabilitation techniques for sports medicine and athletic training. Boston: McGraw-Hill; 2004. https://search.library. wisc.edu/catalog/9910018157502121/cite

[3] Razeghi M, Batt ME. Foot type classification: A critical review of current methods. Gait \& Posture. 2002; 15(3):282-91. [DOI:10.1016/ s0966-6362(01)00151-5]

[4] Periya SN, Alagesan J. Prevalence and incidence of flat foot among Middle East and Asian Population: An Overview. International Journal of Pharmaceutical Science and Health. 2017; 4(7):1-12. https:// www.researchgate.net/publication/319405949_Prevalence_and incidence_of_flat_foot_among_Middle_East_and_Asian_PopulationAn_Overview

[5] Vicenzino B, Franettovich M, McPoil T, Russell T, Skardoon G. Initial effects of anti-pronation tape on the medial longitudinal arch during walking and running. British Journal of Sports Medicine. 2005; 39(12):939-43. [DOI:10.1136/bjsm.2005.019158] [PMID] [PMCID]

[6] Lee MS, Vanore JV, Thomas JL, Catanzariti AR, Kogler G, Kravitz SR, et al. Diagnosis and treatment of adult flatfoot. The Journal of Foot \& Ankle Surgery. 2005; 44(2):78-113. [DOI:10.1053/j. jfas.2004.12.001] [PMID]

[7] Menz HB, Morris ME, Lord SR. Foot and ankle characteristics associated with impaired balance and functional ability in older people. The Journals of Gerontology. Series A, Biological Sciences and Medical Sciences. 2005; 60(12):1546-52. [DOI:10.1093/gerona/60.12.1546] [PMID]

[8] Dahle LK, Mueller MJ, Delitto A, Diamond JE. Visual assessment of foot type and relationship of foot type to lower extremity injury. Journal of Orthopaedic \& Sports Physical Therapy. 1991; 14(2):704. [DOI:10.2519/jospt.1991.14.2.70] [PMID]

[9] Resende RA, Pinheiro LS, Ocarino JM. Effects of foot pronation on the lower limb sagittal plane biomechanics during gait. Gait \& Posture. 2019; 68:130-5. [DOI:10.1016/j.gaitpost.2018.10.025] [PMID]

[10] Hunt AE, Smith RM. Mechanics and control of the flat versus normal foot during the stance phase of walking. Clinical Biomechanics. 2004; 19(4):391-7. [DOI:10.1016/j.clinbiomech.2003.12.010] [PMID]

[11] Cote KP, Brunet ME, II BMG, Shultz SJ. Effects of pronated and supinated foot postures on static and dynamic postural stability. Journal of Athletic Training. 2005; 40(1):41-6. [PMCID]

[12] Gray EG, Basmajian JV. Electromyography and cinematography of leg and foot ("normal" and flat) during walking. The Anatomical Record. 1968; 161(1):1-15. [DOI:10.1002/ar.1091610101] [PMID]

[13] Holmes CF, Wilcox D, Fletcher JP. Effect of a modified, low-dye medial longitudinal arch taping procedure on the subtalar joint neutral position before and after light exercise. Journal of Orthopaedic \& Sports Physical Therapy. 2002; 32(5):194-201. [DOI:10.2519/ jospt.2002.32.5.194] [PMID]
[14] Rodgers MM, Leveau BF. Effectiveness of foot orthotic devices used to modify pronation in runners. Journal of Orthopaedic \& Sports Physical Therapy. 1982; 4(2):86-90. [DOI:10.2519/ jospt.1982.4.2.86] [PMID]

[15] Feltner ME, MacRae H, MacRae PG, Turner NS, Hartman CA, Summers $\mathrm{ML}$, et al. Strength training effects on rearfoot motion in running. Medicine and Science in Sports and Exercise. 1994; 26(8):1021-7. [DOI:10.1249/00005768-199408000-00014] [PMID]

[16] Pires R, Falcari T, Campo AB, Pulcineli BC, Hamill J, Ervilha UF. Using a support vector machine algorithm to classify lower-extremity EMG signals during running shod/unshod with different foot strike patterns. Journal of Applied Biomechanics. 2019; 35(1):8790. [DOI:10.1123/jab.2017-0349] [PMID]

[17] Chalard A, Belle M, Montané E, Marque P, Amarantini D, Gasq D. Impact of the EMG normalization method on muscle activation and the antagonist-agonist co-contraction index during active elbow extension: Practical implications for post-stroke subjects. Journal of Electromyography and Kinesiology. 2020; 51:102403. [DOI:10.1016/j.jelekin.2020.102403] [PMID]

[18] Khandha A, Manal K, Capin J, Wellsandt E, Marmon A, SnyderMackler $L$, et al. High muscle co-contraction does not result in high joint forces during gait in anterior cruciate ligament deficient knees. Journal of Orthopaedic Research. 2019; 37(1):104-12. [DOI:10.1002/jor.24141] [PMID] [PMCID]

[19] Hubley-Kozey C, Deluzio K, Dunbar M. Muscle co-activation patterns during walking in those with severe knee osteoarthritis. Clinical Biomechanics. 2008; 23(1):71-80. [DOI:10.1016/j.clinbiomech.2007.08.019] [PMID]

[20] Diamond LE, Hoang HX, Barrett RS, Loureiro A, Constantinou $M$, Lloyd DG, et al. Individuals with mild-to-moderate hip osteoarthritis walk with lower hip joint contact forces despite higher levels of muscle co-contraction compared to healthy individuals. Osteoarthritis and Cartilage. 2020; 28(7):924-31. [DOI:10.1016/j. joca.2020.04.008] [PMID]

[21] Heiden TL, Lloyd DG, Ackland TR. Knee joint kinematics, kinetics and muscle co-contraction in knee osteoarthritis patient gait. Clinical Biomechanics. 2009; 24(10):833-41. [DOI:10.1016/j.clinbiomech.2009.08.005] [PMID]

[22] Lloyd DG, Buchanan TS. Strategies of muscular support of varus and valgus isometric loads at the human knee. Journal of Biomechanics. 2001; 34(10):1257-67. [DOI:10.1016/s0021 9290(01)00095-1]

[23] Chen M. [Neuromechanical effects of pronated foot on knee joint stability (Korean)] [PhD. Dissertation]. South Korea: Yonsei University; 2011. https://ir.ymlib.yonsei.ac.kr/handle/22282913/136486

[24] Hak L, Houdijk H, Steenbrink F, Mert A, van der Wurff P, Beek PJ, et al. Stepping strategies for regulating gait adaptability and stability. Journal of Biomechanics. 2013; 46(5):905-11. [DOI:10.1016/j. jbiomech.2012.12.017] [PMID]

[25] Marigold DS, Patla AE. Age-related changes in gait for multi-surface terrain. Gait \& Posture. 2008; 27(4):689-96. [DOI:10.1016/j. gaitpost.2007.09.005] [PMID] 
[26] van den Berg ME, Barr CJ, McLoughlin JV, Crotty M. Effect of walking on sand on gait kinematics in individuals with multiple sclerosis. Multiple Sclerosis and Related Disorders. 2017; 16:15-21. [DOI:10.1016/j.msard.2017.05.008] [PMID]

[27] Impellizzeri FM, Rampinini E, Castagna C, Martino F, Fiorini $S$, Wisloff $U$. Effect of plyometric training on sand versus grass on muscle soreness and jumping and sprinting ability in soccer players. British Journal of Sports Medicine. 2008; 42(1):42-6. [DOI:10.1136/bjsm.2007.038497] [PMID]

[28] Jafarnezhadgero A, Fatollahi A, Amirzadeh N, Siahkouhian M, Granacher U. Ground reaction forces and muscle activity while walking on sand versus stable ground in individuals with pronated feet compared with healthy controls. PloS One. 2019; 14(9):e0223219. [DOI:10.1371/journal.pone.0223219] [PMID] [PMCID]

[29] Durai DBJ, Shaju MF. Effect of sand running training on speed among school boys. International Journal of Physical Education, Sports and Health. 2019; 6(3):117-22. https://www.kheljournal.com/archives/?year=2019\&vol=6\&issue $=3 \&$ part $=B \&$ Articleld $=1475$

[30] Zamparo P, Perini R, Orizio C, Sacher M, Ferretti G. The energy cost of walking or running on sand. European Journal of Applied Physiology and Occupational Physiology. 1992; 65(2):183-7. [DOI:10.1007/bf00705078] [PMID]

[31] Pinnington HC, Lloyd DG, Besier TF, Dawson B. Kinematic and electromyography analysis of submaximal differences running on a firm surface compared with soft, dry sand. European Journal of Applied Physiology. 2005; 94(3):242-53. [DOI:10.1007/s00421-005-1323-6] [PMID]

[32] World Medical Association. World Medical Association Declaration of Helsinki. Ethical principles for medical research involving human subjects. Bulletin of the World Health Organization. 2001; 79(4):373-4. [PMCID] [PMID]

[33] Murley GS, Menz HB, Landorf KB. Foot posture influences the electromyographic activity of selected lower limb muscles during gait. Journal of Foot and Ankle Research. 2009; 2:35. [DOI:10.1186/1757-1146-2-35] [PMID] [PMCID]

[34] Powell DW, Long B, Milner CE, Zhang S. Frontal plane multisegment foot kinematics in high-and low-arched females during dynamic loading tasks. Human Movement Science. 2011; 30(1):105-14. [DOI:10.1016/j.humov.2010.08.015] [PMID]

[35] Abe D, Muraki S, Yanagawa K, Fukuoka Y, Niihata S. Changes in EMG characteristics and metabolic energy cost during 90min prolonged running. Gait \& Posture. 2007; 26(4):607-10. [DOI:10.1016/j.gaitpost.2006.12.014] [PMID]

[36] Gardinier ES. The relationship between muscular co-contraction and dynamic knee stiffness in ACL-deficient non-copers (MSc. Thesis)]. Delaware: University of Delaware; 2009. https://udspace.udel.edu/handle/19716/4251\#files-area

[37] Williams DS, McClay IS, Hamill J, Buchanan TS. Lower extremity kinematic and kinetic differences in runners with high and low arches. Journal of Applied Biomechanics. 2001; 17(2):15363. [DOI:10.1123/jab.17.2.153]

[38] Houck JR, Tome JM, Nawoczenski DA. Subtalar neutral position as an offset for a kinematic model of the foot during walking. Gait \& Posture. 2008; 28(1):29-37. [DOI:10.1016/j. gaitpost.2007.09.008] [PMID]
[39] Oatis CA. Kinesiology: The mechanics and pathomechanics of human movement. $2^{\text {th }}$ ed. Philadelphia: Lippincott Williams \& Wilkins; 2009. https://books.google.com/books/about/Kinesiology.html?id=J6juAAAAMAAJ

[40] Chamberlain A, Munro W, Rickard A. Muscle imbalance. In: Porter SB, Tidy NM, editors. Tidys Physiotherapy. $15^{\text {th }}$ ed. Edinburgh: Elsevier; 2013. [DOI:10.1016/b978-0-7020-4344-4.00014-6]

[41] Mizrahi J, Verbitsky O, Isakov E. Fatigue-induced changes in decline running. Clinical Biomechanics. 2001; 16(3):207-12. [DOI:10.1016/s0268-0033(00)00091-7]

[42] Twomey D, McIntosh A. The effects of low arched feet on lower limb gait kinematics in children. The Foot. 2012; 22(2):60-5. [DOI:10.1016/j.foot.2011.11.005] [PMID]

[43] Farahpour N, Jafarnezhad A, Damavandi M, Bakhtiari A, Allard P. Gait ground reaction force characteristics of low back pain patients with pronated foot and able-bodied individuals with and without foot pronation. Journal of Biomechanics. 2016; 49(9):1705-10. [DOI:10.1016/j.jbiomech.2016.03.056] [PMID]

[44] Farahpour N, Jafarnezhadgero A, Allard P, Majlesi M. Muscle activity and kinetics of lower limbs during walking in pronated feet individuals with and without low back pain. Journal of Electromyography and Kinesiology. 2018; 39:35-41. [DOI:10.1016/j.jelekin.2018.01.006] [PMID]

[45] Alavi-Mehr SM, Jafarnezhadgero A, Salari-Esker F, Zago $M$. Acute effect of foot orthoses on frequency domain of ground reaction forces in male children with flexible flatfeet during walking. The Foot. 2018; 37:77-84. [DOI:10.1016/j. foot.2018.05.003] [PMID]

[46] Jafarnezhadgero A, Madadi-Shad M, Alavi-Mehr SM, Granacher $U$. The long-term use of foot orthoses affects walking kinematics and kinetics of children with flexible flat feet: A randomized controlled trial. PloS One. 2018; 13(10):e0205187. [DOI:10.1371/journal.pone.0205187] [PMID] [PMCID]

[47] Jafarnezhadgero A, Alavi-Mehr SM, Granacher U. Effects of anti-pronation shoes on lower limb kinematics and kinetics in female runners with pronated feet: The role of physical fatigue. PloS One. 2019; 14(5):e0216818. [DOI:10.1371/journal. pone.0216818] [PMID] [PMCID]

[48] Jafarnezhadgero AA, Sorkhe E, Oliveira AS. Motion-control shoes help maintaining low loading rate levels during fatiguing running in pronated female runners. Gait \& posture. 2019; 73:65-70. [DOI:10.1016/j.gaitpost.2019.07.133] [PMID]

[49] Jafarnezhadgero A, Sorkhe E, Meamarbashi A. Efficacy of motion control shoes for reducing the frequency response of ground reaction forces in fatigued runners. Journal of $\mathrm{Ad}-$ vanced Sport Technology. 2019; 3(1):8-21. http://jast.uma. ac.ir/article_763_0.html

[50] Madadi-Shad M, Jafarnezhadgero AA, Sheikhalizade H, Dionisio VC. Effect of a corrective exercise program on gait kinetics and muscle activities in older adults with both low back pain and pronated feet: A double-blind, randomized controlled trial. Gait \& Posture. 2020; 76:339-45. [DOI:10.1016/j.gaitpost.2019.12.026] [PMID] 